\title{
Isoflavones, calcium, vitamin D and inulin improve quality of life, sexual function, body composition and metabolic parameters in menopausal women: result from a prospective, randomized, placebo-controlled, parallel-group study
}

\author{
Salvatore Giovanni Vitale ${ }^{1}$, Salvatore Caruso ${ }^{2}$, Agnese Maria Chiara Rapisarda², Stefano Cianci ${ }^{3}$, \\ Antonio $\mathrm{Cianci}^{2}$ \\ 'Unit of Gynecology and Obstetrics, Department of Human Pathology in Adulthood and Childhood "G. Barresi", University of Messina, \\ Messina, Italy \\ ${ }^{2}$ Department of General Surgery and Medical Surgical Specialties, University of Catania, Catania, Italy \\ ${ }^{3}$ Division of Gynecologic Oncology, Department of Obstetrics and Gynecology, Catholic University of the Sacred Heart, Rome, Italy
}

\begin{abstract}
Introduction: Menopause results in metabolic changes that contribute to increase risk of cardiovascular diseases: increase in low density lipoprotein (LDL) and triglycerides and decrease in high density lipoprotein $(\mathrm{HDL})$, weight gain are associated with a correspondent increase in incidence of hypertension and diabetes. The aim of this study was to evaluate the effect of a preparation of isoflavones, calcium vitamin $D$ and inulin in menopausal women.

Material and methods: We performed a prospective, randomized, placebo-controlled, parallel-group study. A total of 50 patients were randomized to receive either oral preparations of isoflavones (40 mg), calcium (500 mg) vitamin D (300 UI) and inulin (3 g) or placebo (control group). Pre- and post-treatment assessment of quality of life and sexual function were performed through Menopause-Specific Quality of Life Questionnaire (MENQOL) and Female Sexual Function Index (FSFI); evaluations of anthropometric indicators, body composition through bioelectrical impedance analyser, lumbar spine and proximal femur T-score and lipid profile were performed.

Results: After 12 months, a significant reduction in MENQOL vasomotor, physical and sexual domain scores $(p<0.05)$ and a significant increase in all FSFI domain scores $(p<0.05)$ were observed in treatment group. Laboratory tests showed significant increase in serum levels of $\mathrm{HDL}(p<0.05)$. No significant changes of lumbar spine and femur neck T-score $(p>0.05)$ were found in the same group.

Conclusions: According to our data analysis, isoflavones, calcium, vitamin D and inulin may exert favourable effects on menopausal symptoms and signs.
\end{abstract}

Key words: menopause, isoflavones, vitamin D, inulin, lipid metabolism.

\section{Introduction}

Life expectancy significantly increased during the last century, thus currently women spend nearly one-third of their life in postmenopausal state [1]. The loss of reproductive capacity may induce not only endocrine changes but also a significant variation in body image and self-esteem. In addition, the experience of climacteric symptoms may negatively affect the sense of physical and mental well-being [2-4]. Menopausal symptoms commonly include hot flushes, night sweats, irritability, poor concentration, joint pains, vaginal dryness, urogenital disorders [58]. Moreover, menopause results in metabolic changes that contribute to increase risk of cardiovascular diseases: increase in Low Density Lipoprotein (LDL) and triglycerides and decrease in high density lipoprotein $(\mathrm{HDL})$, weight gain are associated with a correspondent increase in incidence of hypertension and diabetes $[9,10]$. Oestrogens deficiency leads to an accelerated period of bone loss which can result in osteoporosis, contributing to increase the morbidity [11, 12]. Considering the detrimental role of menopausal symptoms, several compounds were already tested to counteract them and ameliorate postmenopausal quality of life. Thus, this study aimed to evaluate the effect of a preparation of isoflavones, calcium vitamin $D$ and inulin in menopausal women, taking into account clinical (vasomotor disturbances) as well as metabolic (body fat composition, bone depletion, lipid profile) parameters, quality of life and sexual function. 


\section{Material and methods}

This prospective, randomized, placebo-controlled, parallel-group study was performed at the Department of General Surgery and Medical Surgical Specialties, University of Catania (Italy) between February 2015 and October 2016. The study design is in accordance with the Helsinki Declaration, conforms the Committee on Publication Ethics (COPE) guidelines (http://publicationethics.org/) and was approved by the Institutional Review Board (IRB) of the university hospital in which it was performed. Each patient who participated in this study was well informed regarding the procedures that they would undergo and signed a consent form for data collection for research purposes (no remuneration was offered to be included in this study). We enrolled menopausal Italian women who voluntarily accepted to participate in this study and met the following criteria: age between 40 and 60 years, absence of menses for more than 12 months, presence of vasomotor disturbances. We excluded women who in the previous three months received other drugs such as estrogen, estrogen-progestin or other treatments for menopausal symptoms, specific treatment for osteopenia/osteoporosis, treatment with systemic corticosteroids, antidepressants, anticonvulsants and antibiotics. Other exclusion criteria included any medical condition that could influence the results of the study, such as hyperthyroidism and diabetes, chronic inflammatory diseases and other causes of malabsorption, osteoporosis (T-score $<-2.5$ at the femoral neck or lumbar spine), history of pathologic fractures or presence of metabolic bone diseases such as osteomalacia, hyperparathyroidism, Paget, or significant spine abnormalities.

Patients were subjected to a preliminary assessment including obstetric and personal anamnesis as well as gynaecological examination and transvaginal ultrasound. A single operator performed both clinical and instrumental diagnostic procedures. At the stage of recruitment we performed measurement of bone mineral density (BMD) and T-score of lumbar spine and proximal femur by dual-energy X-ray absorptiometry (DEXA), using HOLOGIC Explorer QDR Series. Based on the measured values, we defined normal condition as T-score $>-1$, osteopenia as $-1>$ T-score $>-2.5$, osteoporosis as T-score $<-2.5$, according to the World Health Organization (WHO) criteria [13]. Furthermore, we performed assessment of quality of life and sexual function through evaluation of the Menopause-Specific Quality of Life Questionnaire (MENQOL) [14] and the Female Sexual Function Index (FSFI) $[15,16]$. Evaluated anthropometric indicators were body mass index (BMI) [17] and waist/hip ratio (WHR), used to evaluate the body fat distribution, considered as gynecoid when WHR $<0.8$, and androgenic when WHR $>0.8$ [18]. The evaluation of body composition was assessed by bioelectrical impedance analyser (BIA, Biodynamics 450 model). The fat body mass, lean mass and water distribution were calculated starting from body impedance through a build-in software, taking into account personal information, including weight, height, age, sex. Laboratory tests were performed at the laboratory of the Department of General Surgery and Medical Surgical Specialties of University of Catania. Total cholesterol, HDL, LDL, triglycerides and serum 25-OH-vitamin D levels were evaluated at baseline and after 12 months. Total cholesterol, HDL, triglycerides were quantified by colorimetry while LDL was calculated using the formula of Friedewald, whereas total cholesterol was subtracted from the sum of HDL and triglycerides divided by 5 . Serum 25-OH-vitamin D levels were measured at baseline to assess the state of vitamin D repletion and to subsequently monitor the vitamin D intake.

After the baseline assessment, patients were randomly assigned in a non-stratified $1: 1$ ratio in two groups: group A received an oral dose of isoflavones (40 mg), calcium (500 mg), vitamin D (300 UI), and inulin (3 g); group B (control) received placebo. A computer-generated randomization schedule (www.randomization.com) was used, with concealed allocation of study drugs in consecutively numbered, sealed boxes. Patients and investigators were masked to treatment allocation. Any patients taking less than $80 \%$ of the allocated dose of study drug was regarded as non-compliant. An independent data safety and monitoring committee evaluated the results of the study.

Follow-up was performed at 3, 6 and 12 months, evaluating the same parameters at the baseline. Any adverse reaction was recorded during each follow-up. Student's $t$ test was used to compare all clinical, instrumental and laboratory data obtained at baseline with those obtained on each follow-up. Statistical tests were two-tailed, significance level was set at 5\%. All the design, analysis, interpretation of data, drafting and revisions followed the CONSORT (CONsolidated Standards of Reporting Trials) and SPIRIT (Standard Protocol Items: Recommendation for Interventional Trials) Statements, available through the EQUATOR (Enhancing the QUAlity and Transparency Of health Research) network (http:// www.equator-network.org/).

\section{Results}

We enrolled 50 menopausal Italian women (see CONSORT 2010 flow diagram in Fig. 1) aged 42 to 57 years (mean age $52.6 \pm 3.69$ years), with an average age at menopause onset of $49.28 \pm 3.59$ and an average of $3.32 \pm 1.62$ years since menopause.

Among enrolled women, there weren't any recent history (at least five years before recruitment) of oral contraceptives or hormone replacement therapy.

Baseline anamnestic, clinical and laboratory data regarding the groups of patients on isoflavones and on 


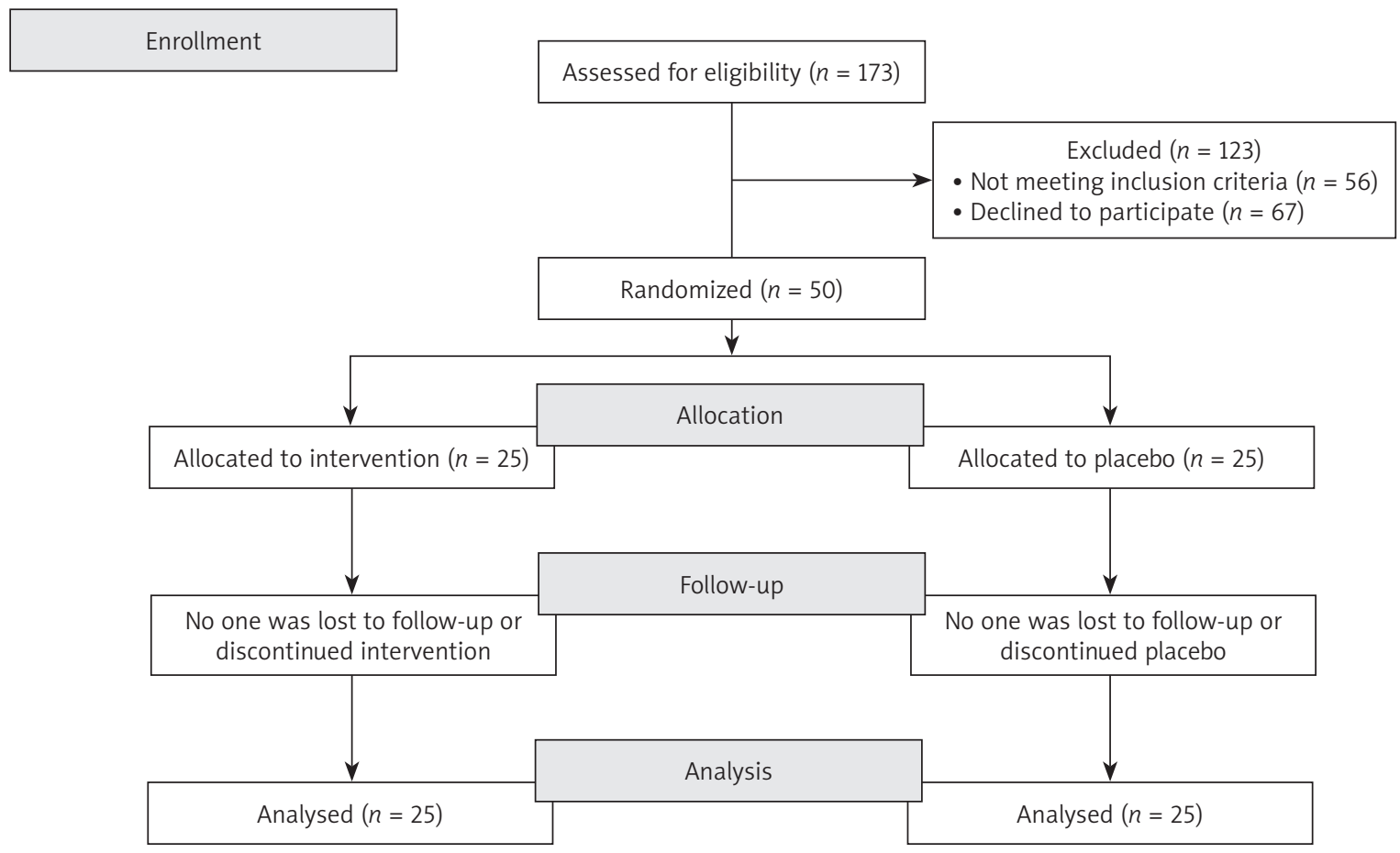

Fig. 1. CONSORT 2010 flow diagram

placebo were statistically compared and are shown in Table 1. The two groups were observed to be homogeneous for age, parity, menopause and others clinical and instrumental analysed parameters with the exception of physical and sexual domains of MENQOL (higher in group A) and desire, orgasm and pain domains of FSFI (higher in group B), allowing further analysis at followup. After 12 months, data were statistically compared with those detected at baseline. In group A, the analysis of MENQOL vasomotor, physical and sexual domains showed a significant reduction of scores $(p<0.05)$; on the other hand, in group $B$ there was a significant increase $(p<0.05)$ in physical and sexual domains while no significant changes in vasomotor domain (Fig. 2). Moreover, no significant variations in psychosocial domain were observed in both groups. In group $A$, the analysis of FSFI showed a significant increase $(p<0.05)$ in all domains and total score, while a significant reduction $(p<0.05)$ in all domains was observed in group B (Fig. 3).

Statistical comparison of laboratory test, femur and lumbar T-scores, anthropometrics and body composition data are reported in Table 2 . In group A, we observed a significant increase of $\mathrm{HDL}(p<0.05)$, without any significant change $(p>0.05)$ of total cholesterol, $\mathrm{LDL}$ and triglycerides. No significant variations were observed in group $\mathrm{B}$.

25-OH-vitamin serum levels D were measured at the 12 months follow-up in each group. Women on supplements showed a significantly higher serum $25-\mathrm{OH}$-vitamin D levels compared to baseline values, while no significant changes were observed in women on placebo.
Regarding anthropometric and body composition indicators, we observed a significant increase of BMI and body fat mass in group $B$, while there were not significant variations in group $A$. Results of femur and lumbar t-scores values indicated no significant changes in group $\mathrm{A}(p>0.05)$, whereas we observed a significant reduction in group $B$ for both values $(p<0.05)$.

Throughout gynaecological follow-up, no significant clinical changes were observed. At transvaginal ultrasound, no significant variations were observed in the endometrial thickness average values. However, four patients (two on isoflavones and two on placebo) manifested a discreet vaginal bleeding, associated in three cases with endometrial thickness greater than $4.0 \mathrm{~mm}$. Subsequently, diagnostic hysteroscopy revealed one case of atrophyc endometrium and three case of endometrial polyp.

Finally, regarding the adverse events, in group A 2 patients reported occurrence of flatulence, 3 reported the onset of constipation and 2 nausea; in group $B$ 2 patients reported onset of nausea and 2 constipation.

\section{Discussion}

Isoflavones have emerged as interesting compounds as they seem able to improve menopausal symptoms without causing significant side effects [1921]. Despite a large number of pubslished studies, their role on women's health still remains debated. This inconsistency may depend, at least in part, by the differences in dosages, type of cohorts and designs among 
Table 1. Comparison of baseline characteristics between group A (treated with isoflavones, calcium, vitamin D and inulin) and group B (placebo)

\begin{tabular}{|c|c|c|c|}
\hline Baseline characteristics & Group A & Group B & $p$ \\
\hline Age (years) & $52.72 \pm 4.02$ & $52.48 \pm 3.4$ & $8.37 \times 10^{-1}$ \\
\hline Menopause (years) & $3.32 \pm 1.38$ & $3.32 \pm 1.86$ & $10.00 \times 10^{-1}$ \\
\hline Age of Menopause (years) & $49.40 \pm 3.65$ & $49.16 \pm 3.60$ & $8.36 \times 10^{-1}$ \\
\hline Parity ( ${ }^{\circ}$ of children) & $1.84 \pm 0.85$ & $1.60 \pm 0.76$ & $2.65 \times 10^{-1}$ \\
\hline $\mathrm{N}^{\circ}$ of vaginal delivery & $1.32 \pm 1.02$ & $1.20 \pm 0.95$ & $6.94 \times 10^{-1}$ \\
\hline $\mathrm{N}^{\circ}$ of caesarean section & $0.52 \pm 0.65$ & $0.40 \pm 0.57$ & $5.42 \times 10^{-1}$ \\
\hline BMI $\left(\mathrm{kg} / \mathrm{m}^{2}\right)$ & $26.10 \pm 4.14$ & $25.67 \pm 4.66$ & $7.20 \times 10^{-1}$ \\
\hline WHR & $0.83 \pm 0.06$ & $0.83 \pm 0.05$ & $8.76 \times 10^{-1}$ \\
\hline Total cholesterol (mg/dl) & $216.96 \pm 19.29$ & $212 \pm 21.79$ & $4.26 \times 10^{-1}$ \\
\hline $\mathrm{HDL}(\mathrm{mg} / \mathrm{dl})$ & $60.12 \pm 7.31$ & $60.16 \pm 7.64$ & $9.83 \times 10^{-1}$ \\
\hline LDL (mg/dl) & $136.976 \pm 22.48$ & $132.624 \pm 21.77$ & $4.82 \times 10^{-1}$ \\
\hline Triglycerides & $88.68 \pm 7.03$ & $89.28 \pm 7.16$ & $4.95 \times 10^{-1}$ \\
\hline Lean mass (kg) & $44.72 \pm 5.67$ & $44.72 \pm 6.49$ & $10.00 \times 10^{-1}$ \\
\hline Fat mass (kg) & $22.164 \pm 6.95$ & $20.32 \pm 8.28$ & $4.13 \times 10^{-1}$ \\
\hline Total body water (I) & $32.57 \pm 3.76$ & $33.10 \pm 5.21$ & $6.47 \times 10^{-1}$ \\
\hline Intracellular water (I) & $16.80 \pm 2.06$ & $18.20 \pm 4.66$ & $1.64 \times 10^{-1}$ \\
\hline Extracellular water (l) & $15.76 \pm 2.39$ & $14.90 \pm 3.19$ & $2.22 \times 10^{-1}$ \\
\hline 25-OH-vit D (ng/ml) & $30.48 \pm 5.15$ & $31.32 \pm 5.46$ & $5.98 \times 10^{-1}$ \\
\hline Lumbar spine T-score (DS) & $-1.648 \pm 0.55$ & $-1.568 \pm 0.55$ & $6.05 \times 10^{-1}$ \\
\hline Femoral neck T-score (DS) & $-0.66 \pm 0.71$ & $-0.556 \pm 0.62$ & $6.00 \times 10^{-1}$ \\
\hline MENQOL domains & Group A & Group B & $p$ \\
\hline Vasomotor & $3.88 \pm 1.98$ & $3.09 \pm 1.20$ & $8.90 \times 10^{-2}$ \\
\hline Psychosocial & $4.01 \pm 1.76$ & $3.09 \pm 1.77$ & $5.30 \times 10^{-2}$ \\
\hline Physic & $3.85 \pm 1.42$ & $2.96 \pm 1.34$ & $1.50 \times 10^{-2}$ \\
\hline Sexual & $5.48 \pm 1.82$ & $4.17 \pm 2.15$ & $2.40 \times 10^{-2}$ \\
\hline FSFI domains & Group A & Group B & $p$ \\
\hline Desire & $2.90 \pm 0.81$ & $3.50 \pm 1.08$ & $2.40 \times 10^{-2}$ \\
\hline Arousal & $3.02 \pm 1.11$ & $3.54 \pm 1.25$ & $1.48 \times 10^{-1}$ \\
\hline Lubrication & $3.20 \pm 1.08$ & $3.85 \pm 1.34$ & $6.00 \times 10^{-2}$ \\
\hline Orgasm & $2.77 \pm 1.23$ & $3.54 \pm 1.23$ & $4.30 \times 10^{-2}$ \\
\hline Satisfaction & $3.97 \pm 1.04$ & $4.48 \pm 1.22$ & $1.40 \times 10^{-1}$ \\
\hline Pain & $2.91 \pm 0.81$ & $3.84 \pm 1.35$ & $5.00 \times 10^{-3}$ \\
\hline Total score & $18.78 \pm 5.24$ & $22.75 \pm 6.54$ & $2.80 \times 10^{-2}$ \\
\hline
\end{tabular}

FSFI - Female Sexual Function Index, MENQOL - Menopause-Specific Quality of Life Questionnaire, BMI - body mass index, WHR - waist-hip ratio; $\mathrm{HDL}$ - high density lipoproteins; LDL - low density lipoproteins

Data are expressed as mean \pm standard deviation

the different studies as well as could be due to different bioavailability between various supplements [22].

Although the published data about this topic are not robust, our results seem to underline that isoflavones together with calcium, vitamin D and inulin improve vasomotor disturbances as well as quality of life and sexual function in menopausal women. Furthermore, this mixture seems to ameliorate lipid profile and body composition toward pre-menopausal homeostatic condition. A meta-analysis of 38 studies confirmed the positive effect of isoflavones on lipid profile, with reduction of LDL and increase of HDL [23]. Furthermore, 


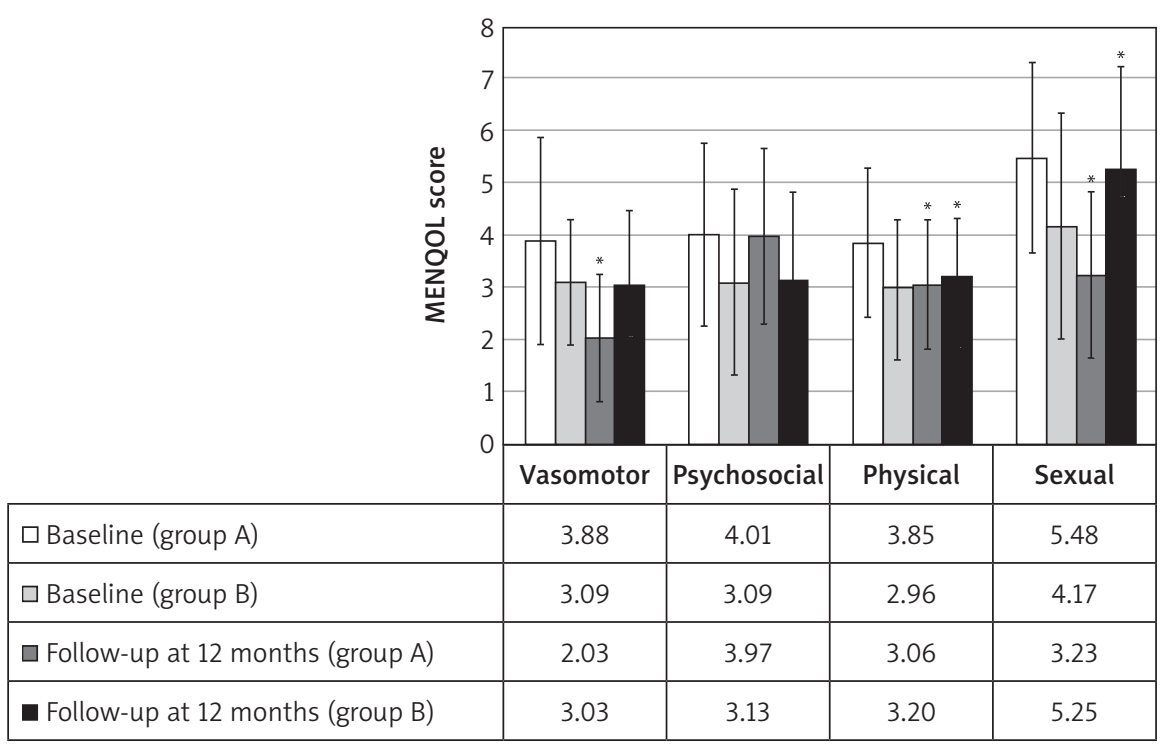

Baseline vs. follow-up 12 months, $p<0.05$

Fig. 2. Menopause-Specific Quality of Life Questionnaire (MENQOL) scores at baseline and at 12-months follow-up

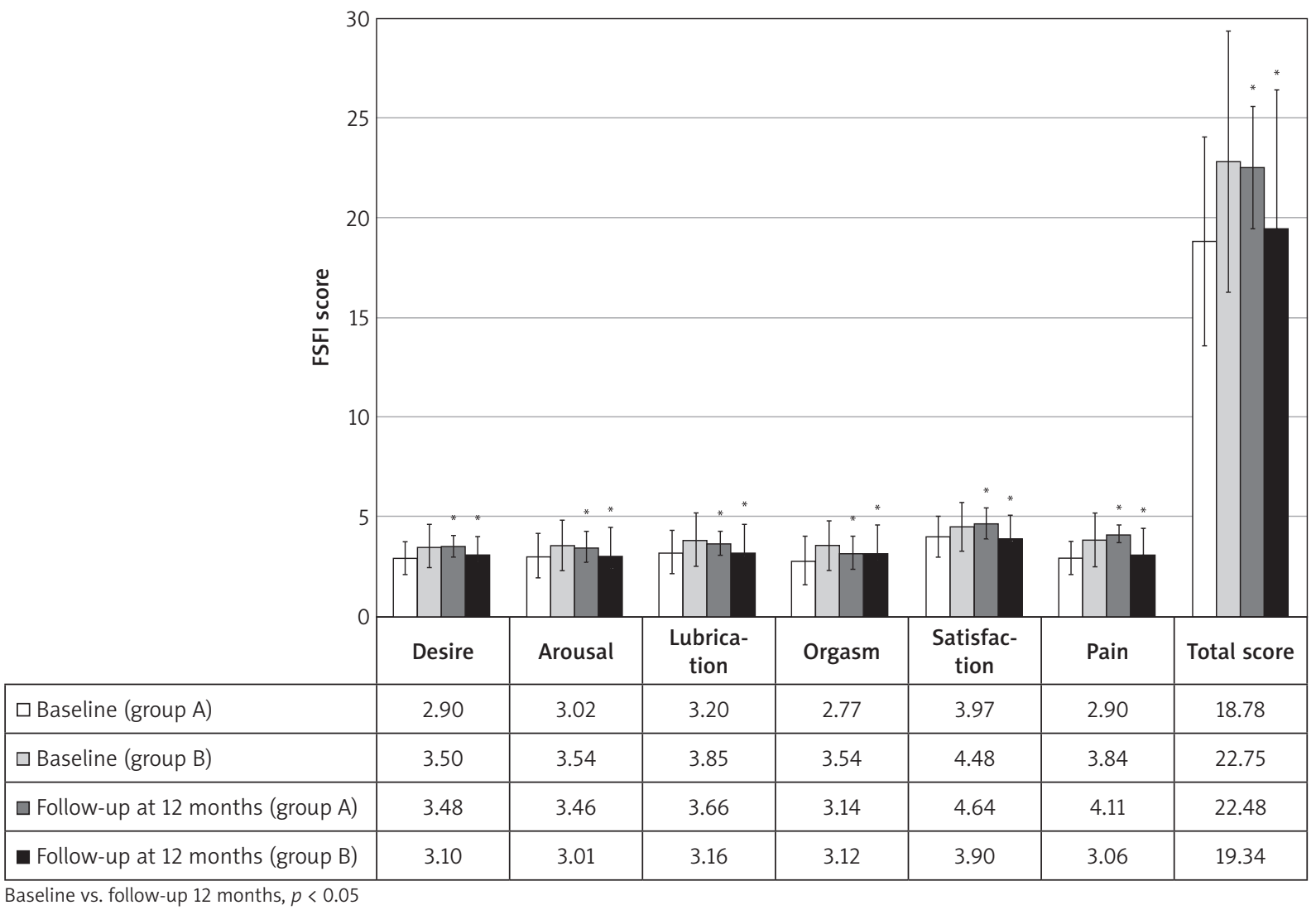

Fig. 3. Female Sexual Function Index (FSFI) scores at baseline and at 12-months follow-up

several observational studies $[24,25]$ reported that a diet high in soy is correlated with lower BMI, waist circumference and body fat mass. In partial agreement with these previous data, we observed a positive effect on HDL level, but no significant effects on others labora- tory parameters and body composition. Finally, we evidenced a preventive effect on bone tissue depletion: according to our data analysis, after 12 months there was a significant reduction of the T-score values in group $B$, while no significant change was shown in group $A$. 
Table 2. Comparison of laboratory tests, femur and lumbar T-scores, anthropometrics and body composition data between baseline and 12-months follow-up in group A (treated with isoflavones, calcium, vitamin D and inulin) and group B (placebo)

\begin{tabular}{|c|c|c|c|}
\hline Group A & & & \\
\hline Parameters & Baseline & 12-months follow-up & $p$ \\
\hline BMI $\left(\mathrm{kg} / \mathrm{m}^{2}\right)$ & $26.10 \pm 4.14$ & $26.13 \pm 4.14$ & $6.56 \times 10^{-1}$ \\
\hline WHR & $0.83 \pm 0.06$ & $0.83 \pm 0.06$ & $3.82 \times 10^{-1}$ \\
\hline Lean mass (kg) & $44.72 \pm 5.67$ & $44.79 \pm 5.78$ & $5.72 \times 10^{-1}$ \\
\hline Fat mass (kg) & $22.16 \pm 6.95$ & $22.17 \pm 6.96$ & $9.51 \times 10^{-1}$ \\
\hline Total body water (l) & $32.57 \pm 3.76$ & $32.43 \pm 3.83$ & $3.29 \times 10^{-1}$ \\
\hline Intracellular water (l) & $16.80 \pm 2.06$ & $16.77 \pm 2.10$ & $6.49 \times 10^{-1}$ \\
\hline Extracellular water (l) & $15.76 \pm 2.39$ & $15.66 \pm 2.42$ & $2.01 \times 10^{-1}$ \\
\hline Total cholesterol (mg/dl) & $216.96 \pm 19.29$ & $215.88 \pm 18.95$ & $1.74 \times 10^{-1}$ \\
\hline $\mathrm{HDL}(\mathrm{mg} / \mathrm{dl})$ & $60.12 \pm 7.31$ & $60.68 \pm 6.87$ & $7.61 \times 10^{-3}$ \\
\hline LDL (mg/dl) & $136.98 \pm 22.48$ & $136.22 \pm 22.29$ & $3.48 \times 10^{-1}$ \\
\hline 25-OH-vit D (ng/ml) & $30.48 \pm 5.15$ & $32.76 \pm 5.32$ & $3.25 \times 10^{-12}$ \\
\hline Triglycerides & $99.32 \pm 14.26$ & $94.88 \pm 15.25$ & $5.99 \times 10^{-2}$ \\
\hline Lumbar spine T-score (DS) & $-1.64 \pm 0.55$ & $-1.40 \pm 1.11$ & $2.35 \times 10^{-1}$ \\
\hline Femoral neck T-score (DS) & $-0.66 \pm 0.71$ & $-0.62 \pm 0.69$ & $5.35 \times 10^{-2}$ \\
\hline \multicolumn{4}{|l|}{ Group B } \\
\hline Parameters & Baseline & 12-months follow-up & $p$ \\
\hline BMI $\left(\mathrm{kg} / \mathrm{m}^{2}\right)$ & $25.67 \pm 4.66$ & $25.86 \pm 4.73$ & $3.63 \times 10^{-3}$ \\
\hline WHR & $0.83 \pm 0.05$ & $0.83 \pm 0.05$ & $5.78 \times 10^{-1}$ \\
\hline Lean mass (kg) & $44.72 \pm 6.49$ & $44.93 \pm 6.60$ & $9.78 \times 10^{-2}$ \\
\hline Fat mass (kg) & $20.32 \pm 8.28$ & $20.51 \pm 8.33$ & $2.50 \times 10^{-2}$ \\
\hline Total body water (l) & $33.10 \pm 5.21$ & $33.27 \pm 5.29$ & $1.49 \times 10^{-1}$ \\
\hline Intracellular water (I) & $18.20 \pm 4.66$ & $18.21 \pm 4.64$ & $7.39 \times 10^{-1}$ \\
\hline Extracellular water (I) & $14.90 \pm 3.19$ & $15.06 \pm 3.17$ & $9.21 \times 10^{-2}$ \\
\hline Total cholesterol (mg/dl) & $212.00 \pm 21.79$ & $213.08 \pm 21.00$ & $5.62 \times 10^{-2}$ \\
\hline $\mathrm{HDL}(\mathrm{mg} / \mathrm{dl})$ & $60.16 \pm 7.64$ & $60.40 \pm 7.47$ & $2.82 \times 10^{-1}$ \\
\hline LDL (mg/dl) & $132.62 \pm 21.77$ & $133.43 \pm 22.02$ & $1.56 \times 10^{-1}$ \\
\hline Triglycerides & $89.28 \pm 7.16$ & $96.24 \pm 10.42$ & $9.31 \times 10^{-1}$ \\
\hline 25-OH-vit D (ng/ml) & $31.32 \pm 5.46$ & $31.36 \pm 6.22$ & $9.40 \times 10^{-1}$ \\
\hline Lumbar spine T-score (DS) & $-1.57 \pm 0.55$ & $-1.66 \pm 0.51$ & $7.94 \times 10^{-5}$ \\
\hline Femoral neck T-score (DS) & $-0.56 \pm 0.62$ & $-0.63 \pm 0.55$ & $1.29 \times 10^{-2}$ \\
\hline
\end{tabular}

BMI - body mass index, WHR - waist-hip ratio, $\mathrm{HDL}$ - high density lipoproteins, LDL - low density lipoproteins Data are expressed as mean \pm standard deviation

The possibility that soy isoflavones and vitamin D may have a synergistic effect on bone metabolism has been already investigated through cell cultures and animal models, although the machinery behind these effect is still unclear [26-31].

\section{Conclusions}

Our data analysis allow us to attest the positive effects of isoflavones together with calcium, vitamin D and inulin on menopausal symptoms, quality of life, sexual function, body composition and metabolic parameters. Nevertheless our results should be considered in the light of several limitations: the described positive effects may depend by just few of the used substances which composed the therapeutic mixture; we are unable to verify whether the positive effects would start from a particular dosage (dose-dependent therapeutic threshold) and/or it is directly related to it (dosage/effects direct proportion); our results are based on a small 
cohort; study design did not allow a direct post-treatment comparison between group $A$ (treated) and group $B$ (placebo) for the analysed parameters.

In this view, we strongly solicit further studies on larger cohorts and with greater statistical power which may accurately define the therapeutic effect(s) of isoflavones together with calcium, vitamin $D$ and inulin in menopausal women.

\section{Disclosure}

Authors report no conflict of interest.

\section{References}

1. Lobo RA, Davis SR, De Villiers TJ, et al. Prevention of diseases after menopause. Climacteric 2014; 17: 540-556.

2. Nappi RE, Lachowsky M. Menopause and sexuality: prevalence of symptoms and impact on quality of life. Maturitas 2009; 63: 138-141.

3. Cianci A, Maiolino L, Giunta G, et al. Neurovegetative disorders of perimenopausal women treated with docosahexaenoic acid (DHA, $625 \mathrm{mg}$ ) Gynecol Endocrinol 2017; 31: 1-5.

4. Pawlak IE, Wolińska W, Mroczek B. Impact of climacteric and depressive symptoms on the quality of life of postmenopausal women. Fam Med Prim Care Rev 2016; 3: 325-331.

5. Dennerstein L, Dudley EC, Hopper JL, et al. A prospective populationbased study of menopausal symptoms. Obstet Gynecol 2000; 96: 351-358

6. Martin KA, Manson JE. Approach to the patient with menopausal symptoms. J Clin Endocrinol Metab 2008; 93: 4567-4575.

7. Caruso S, Cianci S, Vitale SG, et al. Effects of ultralow topic estriol dose on vaginal health and quality of life in postmenopausal women who underwent surgical treatment for pelvic organ prolapse. Menopause 2017; 24: 900-7.

8. Caruso S, Cianci S, Amore FF, et al. Quality of life and sexual function of naturally postmenopausal women on an ultralow-concentration estriol vaginal gel. Menopause 2016; 23: 47-54

9. Leuzzi C, Marzullo R, Modena MG. Is menopause a risk factor for ischemic heart disease in women? G Ital Cardiol (Rome) 2012; 13: 401-406.

10. Carranza-Lira S, Azpilcueta YM, Ortiz SR. Relation between visceral fat and carotid intimal media thickness in Mexican postmenopausal women: a preliminary report. Prz Menopauzalny 2016; 15: 81-84.

11. Lo JC, Burnett-Bowie SA, Finkelstein JS. Bone and the perimenopause. Obstet Gynecol Clin North Am 2011; 38: 503-517.

12. Cyganek A, Pietrzak B, Wielgoś M, Grzechocińska B. Menopause in women with chronic immunosuppressive treatment - how to help those patients. Prz Menopauzalny 2016; 15: 1-5.

13. WHO. Assessment of fracture risk and its application to screening for postmenopausal osteoporosis: technical report series 843. WHO, Geneva 1994.

14. Radtke JV, Terhorst L, Cohen SM. The Menopause-Specific Quality of Life Questionnaire: psychometric evaluation among breast cancer survivors. Menopause 2011; 18: 289-295.

15. Chedraui P, Perez-Lopez FR, San Miguel G, Avila C. Assessment of sexuality among middle-aged women using the Female Sexual Function Index. Climacteric 2009; 12: 213-221.

16. Wiegel M, Meston C, Rosen R. The female sexual function index (FSFI): cross-validation and development of clinical cut off scores. J Sex Marital Ther 2005; 31: 1-20.

17. Keys A, Fidanza F, Karvonen MJ, et al. Indices of relative weight and obesity. J Chronic Dis 1972; 25: 329-343.

18. Van der Kooy K, Seidell JC. Techniques for the measurement of visceral fat: a practical guide. Int J Obes Relat Metab Disord 1993; 17: 187-196.

19. Kupferer EM, Dormire SL, Becker H. Complementary and alternative medicine use for vasomotor symptoms among women who have discontinued hormone therapy. J Obstet Gynecol Neonatal Nurs 2009; 38: 50-59.
20. Cianci A, Colacurci N, Paoletti AM, et al. Soy isoflavones, inulin, calcium, and vitamin D3 in post-menopausal hot flushes: an observational study. Clin Exp Obstet Gynecol 2015; 42: 743-745.

21. Caruso S, Cianci S, Cariola M, et al. Effects of nutraceuticals on quality of life and sexual function of perimenopausal women. J Endocrinol Invest 2017; 40: 27-32.

22. Baber R. Phytoestrogens and post reproductive health. Maturitas 2010; 66: 344-349.

23. Anderson JW, Bush HM. Soy protein effects on serum lipoproteins: a quality assessment and meta-analysis of randomized, controlled studies. J Am Coll Nutr 2011; 30: 79-91.

24. Guthrie JR, Ball M, Murkies A, Dennerstein L. Dietary phytoestrogen intake in mid-life Australian-born women: relationship to health variables. Climacteric 2000; 3: 254-261.

25. Goodman-Gruen D, Kritz-Silverstein D. Usual dietary isoflavone intake and body composition in postmenopausal women. Menopause 2003; 10: 427-432.

26. Park CY, Weaver CM. Vitamin D interactions with soy isoflavones on bone after menopause: a review. Nutrients 2012; 4: 1610-1621.

27. Zhao B, Zhang W, Du S, Zhou Z. Vitamin D receptor Bsml polymorphism and osteoporosis risk in post-menopausal women. Arch Med Sci 2016; 12: $25-30$

28. Colonese F, La Rosa VL, Laganà AS, et al. Comment on: "Is there a role for vitamin D in human reproduction?" Horm Mol Biol Clin Investig 2017; 29: 37-8.

29. Laganà AS, Vitale SG, Ban Frangež H, et al. Vitamin D in human reproduction: the more, the better? An evidence-based critical appraisal. Eur Rev Med Pharmacol Sci 2017; 21: 4243-4251.

30. Aslan N, Demirci K, Güler T, et al. The effect of vitamin D on clinical manifestations and activity of Behçet's disease. Postepy Dermatol Alergol. 2017; 34: 15-20.

31. Pelczyńska M, Grzelak T, Sperling M, et al. Impact of 25-hydroxyvitamin $D$, free and bioavailable fractions of vitamin $D$, and vitamin $D$ binding protein levels on metabolic syndrome components. Arch Med Sci 2017; 13: $745-752$ 